\title{
Veranstaltungen
}

https://doi.org/10.1515/bd-2020-0050

\section{Literatur unter Bäumen - Lesen am See}

\section{Die Open-Air-Bibliothek auf der Landesgartenschau Überlingen vom 23. April bis 18. Oktober}

„Das Paradies habe ich mir immer wie eine Art Bibliothek vorgestellt“, hat der argentinische Schriftsteller Jorge Luis Borges einmal geschrieben. Gewöhnliche Zeitgenossen sehen das Paradies wohl eher als einen herrlichen Garten - mit Bäumen, Wiesen, Blumen, Wasser. Wie auch immer: Bei der diesjährigen Landesgartenschau in Überlingen lassen sich beide Traumbilder aufs schönste vereinen. Von April bis Oktober erwartet das Publikum hier eine einzigartige Szenerie: eine Bibliothek mit über eintausend Werken der Bodensee-Literaturgeschichte - unter freiem Himmel wohlgemerkt.

„Der Bodensee ist eine Landschaft der Literaten“, so das Fazit des Autors und Literaturhistorikers Manfred Bosch in seinem hochgerühmten Standardwerk „Bohème am Bodensee“. Auf überschaubarem Raum sind hier seit rund 1.000 Jahren in den Anrainerländern Deutschland, Österreich, Liechtenstein und der Schweiz literarische Werke von Rang entstanden. Überlingen nimmt dabei eine ganz zentrale Rolle ein. Zahlreiche Literaten waren hier ansässig oder regelmäßig zu Gast, seit mehr als sechs Jahrzehnten wird hier der Bodensee-Literaturpreis verliehen und mit Martin Walser lebt einer der bedeutendsten deutschsprachigen Autoren in der Stadt.

Während der Landesgartenschau können die Besucher der Villengärten direkt am Bodenseeufer unter schattigen alten Bäumen in bequemen Sesseln Platz nehmen, ihre Blicke und Gedanken übers Wasser schweifen lassen und sich in ein Buch vertiefen. In den Containern und Regalen der Open-Air-Bibliothek ist hier eine einmalige Auswahl an Büchern zur Literaturgeschichte der Vierländerregion wetterfest untergebracht - mit Werken von Mönch Walahfrid Strabo aus dem Mittelalter bis hin zu jungen Gegenwartsautoren. Rund 120 Verlage haben die Bücher beigesteuert.

Der Reutlinger Literaturorganisator Peter Reifsteck, am Bodensee durch das literarisch-kulinarische Festival „WortMenue“ seit zwanzig Jahren bekannt, hat das Konzept der Bibliothek gemeinsam mit dem Überlinger Literaturförderer Oswald Burger entwickelt. Studierende des Studiengangs Architektur der Hochschule Konstanz für Technik, Wirtschaft und Gestaltung (HTWG) haben die 
Bibliothek unter Leitung der Professoren Myriam Gautschi und Stefan Krötsch entworfen und mit Unterstützung der Kreishandwerkerschaft gemeinsam umgesetzt. Außergewöhnliche Sitz- und Liegeelemente werden vom Designmöbel-Hersteller Vitra zur Verfügung gestellt. Die Bibliothek wird gefördert durch den IBK-Kleinprojektefonds im Interreg V Programm „Alpenrhein-Bodensee-Hochrhein“.

Begleitet wird die Bibliothek von einer Lesungsreihe mit renommierten Autorinnen und Autoren der Bodenseeregion - jeweils am ersten Dienstag der sechs Veranstaltungsmonate. Mit dabei sind Arnold Stadler, Eva Gesine Baur, Bruno Epple, Verena Roßbacher und Peter Stamm. Gleichzeitig erinnert die Reihe aber auch an bereits verstorbene Dichter vom See wie Maria Beig, Jacob Picard, Annette von Droste-Hülshoff, Franz Michael Felder und Markus Werner. Diese werden vorgestellt und gelesen von den Publizisten Irene Ferchl, Anne Overlack und Oswald Burger, der Schauspielerin Dorothea Neukirchen und Jürgen Thaler, dem Leiter des Franz-Michael-Felder-Archivs der Vorarlberger Landesbibliothek. Ausführliche Informationen zum Lesungsprogramm finden sich auf der Homepage der Landesgartenschau Überlingen: https://www.ueberlingen 2020.de/de.

\section{Das Veranstaltungsprogramm im Überblick}

\section{Dienstag, 5. Mai}

11 Uhr | Open-Air-Bibliothek auf der LGS Überlingen

Lesen am See: 10 Stunden, 10 Vorleserinnen \& Leser: Mein Lieblingsbuch vom Bodensee

Moderation: Oswald Burger

\section{Dienstag, 2. Juni}

19 Uhr | Grüner Salon auf der LGS Überlingen

Lesen am See: Bruno Epple trifft Jacob Picard.

Mit Bruno Epple und Anne Overlack. Moderation: Oswald Burger

\section{Dienstag, 7. Juli}

19 Uhr | Grüner Salon auf der LGS Überlingen

Lesen am See: Peter Stamm trifft Markus Werner.

Mit Peter Stamm und Oswald Burger. 


\section{Dienstag, 4. August}

19 Uhr | Grüner Salon auf der LGS Überlingen

Lesen am See: Eva Gesine Baur trifft Annette von Droste-Hülshoff.

Mit Eva Gesine Baur und Irene Ferchl. Moderation: Oswald Burger

\section{Dienstag, 1. September}

19 Uhr | Grüner Salon auf der LGS Überlingen

Lesen am See: Arnold Stadler trifft Maria Beig.

Mit Arnold Stadler und Schauspielerin Dorothea Neukirchen. Moderation: Oswald Burger

\section{Dienstag, 6. Oktober}

19 Uhr | Grüner Salon auf der LGS Überlingen

Lesen am See: Verena Roßbacher trifft Franz Michael Felder.

Mit Verena Roßbacher und Jürgen Thaler. Moderation: Oswald Burger

Die Open-Air-Bibliothek und das begleitende Literaturprogramm werden gefördert durch den IBK-Kleinprojektefonds im Interreg V Programm „Alpenrhein-Bodensee-Hochrhein“.

\section{Kontakt:}

Peter Reifsteck

Organisationsbüro Open-Air-Bibliothek auf der Landesgartenschau Überlingen Grafeneck 10, D-72770 Reutlingen

Mobil: 0049-171-2013566, E-Mail: mail@reifsteck-literaturbuero.de

\section{Einladung: Fachtagung „Zukunft wissenschaftlicher Bibliotheken?!“}

ZB MED - Informationszentrum Lebenswissenschaften und das Fachmagazin Open Password hatten geplant, im Januar die Fachtagung „Zukunft wissenschaftlicher Bibliotheken?!“ zu veranstalten. Am Tag vorher mussten wir die Tagung wegen eines Bombenfundes in unmittelbarer Nähe zum Veranstaltungsort - verbunden mit Absperrung und Evakuierung - schweren Herzens absagen. Schon mit der Absage haben wir versprochen: Aufgeschoben ist nicht aufgebhoben daher laden wir Sie nun erneut herzlich ein! Die Fachtagung findet am Montag, 
den 18. Mai 2020 am Kölner Standort von ZB MED statt. Am Programm und den Beteiligten hat sich nichts geändert.

Die Veranstaltung geht der Frage nach, ob wissenschaftliche Bibliotheken eine Zukunft haben und gibt direkt die Antwort: In der Keynote erörtert Dr. Dietrich Nelle, ehemaliger Interimsdirektor von ZB MED, die Perspektiven wissenschaftlicher Bibliotheken und zeigt Lösungsmöglichkeiten für die anstehenden Herausforderungen auf.

In mehreren Impulsvorträgen geben Expertinnen und Experten aus unterschiedlichen Blickwinkeln Anregungen und stellen zukunftsweisende Projekte und Entwicklungen vor. Anne Christensen, ehemalige Bibliotheksdirektorin, jetzt Beraterin und Mitinhaberin bei effective WEBWORK, spricht über Strategien für digitale Dienste; ihre Thesen zur Zukunft wissenschaftlicher Bibliotheken bilden die Grundlage für den Beitrag. Prof. Dr. Dietrich Rebholz-Schuhmann, Wissenschaftlicher Direktor von ZB MED, zeigt auf, dass sich Forschung und Bibliothek innerhalb einer Einrichtung gegenseitig ergänzen und auf Augenhöhe zusammenarbeiten. Prof. Dr. Sören Auer, Direktor der TIB - Leibniz-Informationszentrum Technik und Naturwissenschaften, erläutert den Transformationsprozess wissenschaftlicher Bibliotheken von dokumentenbasierten zu wissensbasierten Informationsflüssen und schildert, welche Schritte seine Einrichtung bereits in diese Richtung unternommen hat. Prof. Dr. Konrad Förstner, bei ZB MED Leiter der Informationsdienste und zugleich Professor an der TH Köln, thematisiert die Qualifizierungen für zukünftige Anforderungen in Bibliotheken und stellt gemeinsam mit Marvin Lanczek vom ZBIW den neuen Zertifikatskurs zum „Data Librarian“ vor. Die abschließende Diskussion beleuchtet die Rolle der wissenschaftlichen Bibliotheken als unverzichtbarer Kooperationspartner der Wissenschaft.

Die Fachtagung schließt an das 2019 erschienene Buch „Zukunft der Informationswissenschaft - Hat die Informationswissenschaft eine Zukunft?“ an. Eine erste Veranstaltung am 5. September 2019 an der TU Berlin nahm die Zukunft der Informationswissenschaft in den Fokus. Da das Buch als zentrales Thema auch die Perspektiven wissenschaftlicher Bibliotheken beleuchtet, folgt nun auch zu diesem Schwerpunkt eine Veranstaltung. Bibliotheken stehen ebenso wie ihre Klientel an den Hochschulen und Forschungseinrichtungen vor großen Herausforderungen. Open Access, Forschungsdatenmanagement, Informations- und Datenkompetenz, Langzeitarchivierung, Künstliche Intelligenz, Citizen Science, Personalgewinnung als neuer Engpass, die Anpassung der bibliothekswissenschaftlichen Lehre an neue Anforderungen - um nur diese $\mathrm{zu}$ nennen. Einige wissenschaftliche Bibliotheken wie zum Beispiel ZB MED begegnen dem mit der Intensivierung ihrer Forschung und stehen dadurch in einem bedeutenden Strukturwandel. Dr. Willi Bredemeier, der das Buch zur 
Zukunft der Informationswissenschaft mit zahlreichen Fachbeiträgen renommierter Expertinnen und Experten herausgegeben hat, gibt bei der Fachtagung „Zukunft wissenschaftlicher Bibliotheken?!“ ein Statement zum aktuellen Stand der Debatte.

\section{Alle Infos auf einen Blick:}

- Was: Fachtagung „Zukunft wissenschaftlicher Bibliotheken?!“

- Wer: veranstaltet von ZB MED - Informationszentrum Lebenswissenschaften und Open Password

- Wann: Montag, 18. Mai 2020, ab 14.00 Uhr

- Wo: ZB MED, Standort Köln, Gleueler Straße 60, 50931 Köln

\section{Programm:}

14.00 Uhr Registrierung - Meet and Greet

14.30 Uhr Willkommen!

Gabriele Herrmann-Krotz, Kaufmännisch-administrative Geschäftsführerin $Z B M E D$

14.35 Uhr Statement: Die Debatte geht weiter - zum Buch „Zukunft der Informationswissenschaft“"

Dr. Willi Bredemeier, Herausgeber

14.45 Uhr Keynote: Herausforderungen, Lösungen und Perspektiven wissenschaftlicher Bibliotheken

Dr. Dietrich Nelle, BMBF, ehemaliger Interimsdirektor ZB MED

15.15 Uhr Impulsreferat: Digitale Dienste in Bibliotheken - Thesen für erfolgreiche Strategien

Anne Christensen, effective WEBWORK

15.30 Uhr Impulsreferat: Das forschende Informationszentrum und seine lebenswissenschaftliche Bibliothek auf Augenhöhe

Prof. Dr. Dietrich Rebholz-Schuhmann, ZB MED

15.45 Uhr Impulsreferat: Von dokumentenbasierten zu wissensbasierten Informationsflüssen - Die wissenschaftlichen Bibliotheken im Transformationsprozess

Prof. Dr. Sören Auer, Technische Informationsbibliothek Hannover

16.00 Uhr Impulsreferat: Qualifizierung für die Anforderungen von morgen Ausbildung zum Data Librarian

Prof. Dr. Konrad Förstner, ZB MED und Institut für Informationswissenschaft der TH Köln

Marvin Lanczek, Zentrum für Bibliotheks- und Informationswissenschaftliche Weiterbildung 
16.15 Uhr Diskussion unter Einbeziehung des Plenums: Die wissenschaftlichen Bibliotheken als unverzichtbarer Kooperationspartner der Wissenschaft

Mit Sören Auer, Anne Christensen, Dietrich Nelle und Dietrich Rebholz-Schuhmann

Moderation: Konrad Förstner

17.00 Uhr Empfang

Wir freuen uns, Sie in Köln zu begrüßen! Für unsere Planung möchten wir Sie bitten, sich bis zum 11. Mai 2020 zur Fachtagung anzumelden. Die Teilnahme ist kostenlos.

\title{
Weitere Informationen:
}

- Zum Programm: https://www.zbmed.de/fileadmin/user_upload/NewsBilder/Datei-Anhaenge/2020-05-18_Programm_Zukunft_wissenschaftlicher_ Bibliotheken.pdf

- Zur Anmeldung: http://survey.2ask.de/be37ac414a1398a3/survey.html

„Zukunft der Informationswissenschaft - Hat die Informationswissenschaft eine Zukunft? Grundlagen und Perspektiven - Angebote in der Lehre - An den Fronten der Informationswissenschaft", herausgegeben von Willi Bredemeier - Simon Verlag für Bibliothekswissen, ISBN 978-3-945610-46-6, Berlin 2019.

\section{AGMB 2020: Innovativ in die nächsten 50 Jahre}

\author{
Herzliche Einladung zur AGMB-Jahrestagung mit \\ Firmenausstellung vom 21. - 23.09.2020 in Würzburg
}

Die Tagung der Arbeitsgemeinschaft für Medizinisches Bibliothekswesen e.V. findet in diesem Jahr von Montag, den 21.09. bis Mittwoch, den 23.09.2020 im zentralen Hörsaal- und Seminargebäude Z6 der Universität Würzburg statt.

Sie steht unter dem Motto „AGMB 2020: Innovativ in die nächsten 50 Jahre“ und soll Vorträge, Treffpunkte und Poster $\mathrm{zu}$ folgenden Themenschwerpunkten beinhalten:

- Open Access und Open Science

- Innovative Entwicklungen für Bibliotheksdienstleistungen 
- Evidenzbasierte Medizin und ihre Herausforderungen

- Strategien der Serviceentwicklung und Qualitätssicherung

\section{Informationen im Internet:}

- Einladung und Informationen für Firmen und Sponsoren / Invitation and Information for Companies and Sponsors: https://www.agmb.de/de_ DE/2020-einladung-und-informationen

- Call for Proposals / Poster: http://media02.culturebase.org/data/docs-agmb/ CfP_2020.pdf

- Homepage der AGMB: https://www.agmb.de/de_DE/2020-wuerzburgstartseite

\section{Tagungsort:}

Zentrales Hörsaal- und Seminargebäude Z6 der Universität Würzburg

Am Hubland

D-97074 Würzburg

\section{Kontakt:}

Mit allgemeinen Fragen wenden Sie sich bitte an:

Dr. Iris Reimann, E-Mail: reimann@ub.rwth-aachen.de

\section{Ansprechpartnerin für Firmen}

Frau Almut Wenk, E-Mail: agmb2020@bibliothek.uni-wuerzburg.de Tel.: +49 9313183516 